\title{
The Performance of Analytical Approximations for the Computation of Asian Quanto-Basket Option Prices
}

\author{
Jean-Yves Datey \\ Comission Scolaire de Montréal, Canada \\ Geneviève Gauthier \\ HEC Montréal, Canada \\ Jean-Guy Simonato \\ HEC Montréal, Canada
}

An option contract now commonly encountered is the Asian quanto-basket option. This contract is useful for risk managers willing to participate to the return of an industrial sector with an international exposure without the foreign exchange risk exposition. Although the price of such contracts can be obtained very accurately using Monte Carlo simulation, market participants prefer faster but less accurate analytical approximations. This paper thus examines the precision of three different analytical approximations available to price Asian quanto-basket options. The results of a comprehensive simulation experiment performed on a large test pool of option contracts reveal that the approximations based on the reciprocal gamma and Johnson-type densities are in general the most accurate.

Keywords: analytical approximation, Asian option, basket option, option pricing, quanto option.

*Gauthier and Simonato acknowledge the financial support from the Social Sciences and Humanities Research Council of Canada (SSHRC), Les Fonds pour la Formation de Chercheurs et l'Aide à la Recherche du Québec (FCAR) and the Natural Science and Engineering Research Concil of Canada (NSERC).

(Multinational Finance Journal, 2003, vol. 7, no. $1 \& 2$, pp. 55-82)

(C) Multinational Finance Society, a nonprofit corporation. All rights reserved.

DOI: $10.17578 / 7-1 / 2-3$ 


\section{Introduction}

The world of derivative securities is a constant source of innovation. This has been particularly true since the beginning of the 1980's. The globalization of economies and the increased attention paid to risk management by both financial and non-financial institutions are important factors behind this evolution. Within this context, the instruments proposed to manage the risks associated with different economic factors have been steadily increasing in number as well as in complexity. This is why more complex option contracts known as exotic options were developed during the 80's.

With these new option contracts comes the basic problem of having to price them. Because of their particular characteristics, the pricing formula proposed by Black and Scholes (1973) is inadequate. In such cases, adjustments and generalizations are necessary. This is how various techniques and approximations came to be developed for a large number of exotic options, including Asian options, basket options, barrier options, lookback options, quanto options and others. In this study we examine a type of option contract that is somewhat more complex than those mentioned above: the Asian quanto-basket option (AQB hereafter). In a multinational setting, this contract is useful since it allows risk managers to participate to the returns of a sector with an international exposure without being affected by the foreign exchange risk. This contract thus simplifies the hedging procedures designed to cover the risk associated with a multinational industrial factor since the exchange risk is eliminated by the quanto feature of the contract.

Because of the arithmetic Asian and basket features of this contract, the standard lognormality assumption leads to an expression that cannot be analytically assessed. Indeed, both features imply the sum of lognormal random variables, which is clearly not lognormal. In the literature, several approaches have been proposed to compute the price of option contracts whose payoffs depend on the sum of lognormal variables. For example, for Asian options, we find Monte Carlo simulation (Kemna and Vorst [1990], Fu, Madan and Wang [1996]), lattice and PDE approaches (Hull and White [1993], Vecer [2001]), pseudo-analytic characterizations (Geman and Yor [1993], Dufresne [2000], Bakshi and Madan [2002]), pricing bounds (Rogers and Shi [1995], Nielsen and Sandmann [2002]) and, finally, analytical approximations (Turnbull and Wakeman [1991], Levy [1992], Vorst 
[1992], Milevsky and Posner [1998a], Posner and Milevsky [1998]). Similarly, for the case of basket options we also find Monte Carlo simulation (Joy, Boyle and Tan [1996]), binomial trees (Rubinstein (1994)) and analytical approximations (Gentle [1993], Milevsky and Posner [1998b]).

There are thus a variety of approaches that can be used to determine the value of such options. Those working in the financial industry however, prefer using an approximate analytical solution rather than a more accurate solution involving lengthy numerical calculations that are often incompatible with their integrated computer and risk management systems. Indeed, the risk management process often requires the ability to price a large book of options quickly. In this context, the precision of these approximations becomes an important issue. The main contribution of this study is thus to comprehensively explore and compare the different analytical approximations available for computing the prices of AQB options.

Although some studies have examined the precision of such analytical approximations for either the Asian or basket option cases, a small set of parameter values is typically used to make the comparisons. A more comprehensive study focusing on a large set of the possible parameter values would thus be welcome since the quality of an approximation is not always constant through out the parameter space. It is also important to point out that none of these studies has yet focused on the quality of these approximations for an option contract with both the Asian and basket features. It is not clear that the results found for the Asian or basket case directly apply to AQB case. Indeed, although the Asian and basket options are closely related because of the need to average random variables with lognormal distributions, they show some differences in the correlation structure of the elements being averaged. As discussed in Milevsky and Posner (1998b), the correlation structure can have an impact on the quality of an analytical approximation. Mixing both correlation structures can thus influence the precision of these pricing approaches. Finally, since the option contract examined here also shows a quanto feature which affects the drift of the risk-neutral process of the underlying securities, it uncertain how an approximation such as the one proposed in Milevsky and Posner (1998a) will perform since this approach is sensitive to the sign of the drift.

Three simple analytical approximations which can be computed in 
fractions of a second on a standard desktop computer will be examined. These approaches use approximations of the true unknown risk neutral density and were developed and applied to problems similar to ours by Turnbull and Wakeman (1991), Milevsky and Posner (1998a) and Posner and Milevsky (1998) with the use of a lognormal, reciprocal gamma and Johnson distributions respectively. ${ }^{1}$ We exclude from this comparison the pricing bound approaches that have been developed in the Asian option pricing literature (see Rogers and Shi [1995], Curran [1994] and Nielsen and Sandmann [2002]). These bounds, which can be computed quickly, are usually found to be fairly accurate for Asian options. However, adapting these to the AQB framework is a non-trivial task that is beyond the scope of this paper.

In the next section, the AQB option is described in more details. Section III will examine the risk neutral pricing model, while Section IV will derive and examine the different analytical approximations associated with the use of the pricing model. Section V will present and discuss the results of a simulation study examining the performance of the analytical approximations for computing the prices and hedge parameters. The main results from this study reveal that the two approximations developed in Milevsky and Posner (1999) are by far the most accurate.

\section{The Asian quanto-basket option}

Below we will examine different types of option contracts, the understanding of which will help shed some light on the nature of a quanto-basket Asian option. Unless otherwise specified, we will suppose that the call options being discussed are European-style options, that they lead to a cash payment on the date of expiry and that their underlying assets are composed of equity shares. In what follows, $S_{t}^{(1)}$, $\ldots, S_{t}^{(n)}$ indicate the prices at time $t$ of $n$ different domestic or foreign shares. Note that $S_{t}^{(j)}$ is expressed in currency $j$.

1. Two other approximations obtained by replacing the arithmetic mean with a geometric mean and an adjusted strike price, as done by Vorst (1992) for Asian options and by Gentle (1993) for basket options, have also been examined in an earlier version of this paper. However, because these methods were found to be inferior, they were simply discarded from this version. 
Standard option: This option gives its holder the right to acquire share $j$ on a specific date for a predetermined price $K$. Its payoff function is given by:

$$
\text { Payoff at maturity }=\max \left(S_{T}^{(j)}-K ; 0\right)
$$

and is expressed in currency $j$.

-Arithmetic Asian option: The difference between a standard option and an Asian option is that the latter uses the average value of the underlying assets at predetermined dates $t_{1}, \ldots, t_{m}$ rather than the value upon expiry to calculate the payment amount, this being illustrated as follows:

$$
\text { Payoff atmaturity }=\max \left(\frac{1}{m} \sum_{i=1}^{m} S_{t_{1}}^{(j)}-K ; 0\right),
$$

and is expressed in currency $j$.

Several variations of the Asian option are described in the literature. These are assessed in terms of mean average, geometric mean, discrete mean or continuous mean, and also in terms of average underlying value or average exercise price. In practice however, a few exceptions left aside, the mean is a discrete arithmetic average applied to the underlying value. Throughout this article, this last definition will be used. Utilizing a mean in the payment function protects the holder (or the writer) from being exposed to the risk posed by ad hoc fluctuations of the underlying assets. The mean was introduced on financial markets to protect the different parties from stock market manipulation of underlying values on or near the expiry date of options.

Basket option: A basket option can be seen as a standard option with an underlying composed of several financial assets, all generally expressed in the same currency. This underlying is called a basket. Its payoff function is written as:

$$
\text { Payoff atmaturity }=\max \left(\sum_{j=1}^{n} w_{j} S_{T}^{(j)}-K ; 0\right),
$$


where $w_{1}, \ldots, w_{n}$ are the weights associated with each asset. One of the main advantages of the basket option is that it allows the coverage of several underlying assets at a lesser cost than that of purchasing an option for each underlying asset to be covered.

Quanto option: This variety of option is defined in terms of an underlying made out in a currency other than the currency of the payment to be made upon expiry, as per the following:

$$
\text { Payoff atmaturity }=C_{0}^{(j)} \max \left(S_{T}^{(j)}-X ; 0\right)
$$

where $C_{0}^{(j)}$ represents the number of units of local currency by unit of foreign currency at time $t=0$. For the investor, the quanto option allows indirect investment in a foreign security without being exposed to the foreign exchange risk. It is also a useful tool for risk managers who must cover themselves against potential fluctuations in foreign assets.

Asian quanto-basket option: This option combines the properties of the Asian option, the basket option and the quanto option. Its payment function is as follows:

$$
\text { Payoff atmaturity }=\max \left(\frac{1}{m} \sum_{i=1}^{m} \sum_{j=1}^{n} w_{j} S_{i}^{(j)}-K ; 0\right),
$$

the total amount paid in a predetermined currency. The fixed exchange rates are included in the weights. The same as the other types of option, the AQB option can be a useful tool for the investor as well as the risk manager. For example, an investor wanting to participate in the yield of a particular economic sector with international exposure, but not wanting to be subjected to the currency risk, may be interested in this type of option. The basket would include shares from the main companies involved in the industry across the world, and the payment upon expiry would be made in the investor's local currency. The Asian characteristic of the option protects the investor against possible ad hoc yield fluctuations within the industry. The following example more clearly illustrates the dynamics of this option. 
TABLE 1. Asian Quanto-basket Option Data

\begin{tabular}{lllll}
\hline & & \multicolumn{3}{c}{ Price } \\
\cline { 3 - 5 } Security & weight & date 1 & date 2 & date 3 \\
\hline $\begin{array}{l}\text { Boeing } \\
\text { (United States) }\end{array}$ & $25 \%$ & 55 US\$ & 60 US\$ & 65 US\$ \\
$\begin{array}{l}\text { Bombardier } \\
\text { (Canada) }\end{array}$ & $35 \%$ & 18 CAN\$ & 26 CAN\$ & 23 CAN\$ \\
$\begin{array}{l}\text { Embraer } \\
\text { (Brasil) }\end{array}$ & $40 \%$ & 12 R\$ & $10 \mathrm{R} \$$ & 15 R\$ \\
Basket & $100 \%$ & $\begin{array}{l}24.85 \\
\text { (payoff } \\
\text { currency) }\end{array}$ & $\begin{array}{l}28.10 \\
\text { (payoff } \\
\text { currency) }\end{array}$ & $\begin{array}{l}\text { (payoff } \\
\text { currency) }\end{array}$ \\
\hline
\end{tabular}

Example: an Asian quanto-basket option (CAN\$) related to the aircraft industry. The basket will include shares from the three largest firms in the North American civil aircraft industry. To simplify our example, only three dates were used to calculate the mean. The data pertaining to the example with three currencies are shown in Table 1. Applying these figures to date 1 gives a weighted average of $0.25 \times 55+0.35 \times 18+$ $0.4 \times 12=24.85$ CAN\$. Similar computations for date two and three obtain 28.10 and 30.30 CAN\$. Using an exercise price of 26.50 CAN\$ gives the following payment upon expiry:

Payoff at maturity $=\max \left(\frac{24.85+28.10+30.30}{3}-26.50 ; 0\right) \$ \mathrm{CAN}$.

The following section will develop a theoretical pricing model for these options.

\section{The Theoretical Pricing Framework}

To obtain a pricing model, the methodology suggested in the works of Harrison and Pliska (1981) will be used. The objective of such an approach is to assess the expected value of the option upon expiry using a risk neutral probability measure, and then capitalize it at the risk-free rate. The model supposes the existence of $n$ foreign shares, $n$ exchange 
rates, $n$ risk-free foreign bonds and one risk-free domestic bond, noted as follows:

$$
\begin{gathered}
S^{(j)}=\left\{S_{t}^{(j)}: t \geq 0\right\} \text { for the foreign share } j, \\
C^{(j)}=\left\{C_{t}^{(j)}: t \geq 0\right\} \text { for the exchange rate } j \text { (domestic/foreign), } \\
B^{(j)}=\left\{B_{t}^{(j)}: t \geq 0\right\} \text { for the foreign risk free bond } j \\
D=\left\{D_{t}: t \geq 0\right\} \text { for the domestic risk free bond. }
\end{gathered}
$$

We also assume that lognormal models are applied to the shares and the exchange rates. We will thus apply the following hypothesis to the stochastic processes of these variables:

$$
\begin{gathered}
d S_{t}^{(j)}=H_{j} S_{t}^{(j)} d t+\sigma_{j} S_{t}^{(j)} d W_{t}^{(j)}, j=1, \ldots, n, \\
d C_{t}^{(j)}=v_{j} C_{t}^{(j)} d t+\alpha_{j} C_{t}^{(j)} d Z_{t}^{(j)}, j=1, \ldots, n, \\
d B_{t}^{(j)}=r_{j} B_{t}^{(j)} d t, j=1, \ldots, n, \\
d D_{i}=r D_{i} d t
\end{gathered}
$$

where $W^{(1)}, \ldots, W^{(n)}, Z^{(1)}, \ldots, Z^{(n)}$ are standard Brownian motions with correlations

$$
\begin{aligned}
& \operatorname{Corr}^{P}\left(W_{t}^{(k)}, W_{t}^{(j)}\right)=\theta_{k, j}, \\
& \operatorname{Corr}^{P}\left(Z_{t}^{(k)}, Z_{t}^{(j)}\right)=\lambda_{k, j}, \\
& \operatorname{Corr}^{P}\left(W_{t}^{(k)}, Z_{t}^{(j)}\right)=\rho_{k, j} .
\end{aligned}
$$

We also suppose that share $j$ pays a dividend (possibly nil) on a continuous basis and at a constant rate of $\delta_{j}$. Finally, each of these stochastic processes is built on probability space $(\Omega, F, \mathrm{~F}, P)$ where $\mathrm{F}$ is the filtration $\left\{F_{t}: t \geq 0\right\}$, with: 


$$
F_{t}=\sigma\left(\aleph, Z_{s}^{(j)} \text { and } W_{s}^{(j)}: 0 \leq s \leq t \text { and } j \in\{1,2, \ldots n\}\right)
$$

and $\$$ the set of zero probability events. This represents a standard model that is widely used for this type of problem, but nevertheless entails certain weaknesses, including constant rates of interest and volatilities. The main advantage of this type of model is that it allows practitioners to arrive at an acceptable and usable solution that is realistic enough in terms of empirical observation. If we consider the fact that an option expires within a relatively short period of time, the above-mentioned weaknesses lose a lot of their potential influence.

To find a formula for the price of the option, we need to represent the price process of the underlying stock using a risk neutral probability measure that we will refer to as $Q$. After several manipulations, the details of which are available upon request, we found that this risk neutral measure is unique and that the required process is given by the following stochastic differential equation (SDE):

$$
\begin{gathered}
d S_{t}^{(j)}=\left(\gamma_{j}-\delta_{j}-\alpha_{j} \sigma_{j} \rho_{j, j}\right) S_{t}^{(j)} d t \\
+\sigma_{j} S_{t}^{(j)} d W_{t}^{(j)}, j=1, \ldots, n
\end{gathered}
$$

where $-\alpha_{j} \sigma_{j} \rho_{j, j}$ is an adjustment term for the quanto feature of the option and $\tilde{W}^{(n)}, \ldots, \tilde{W}^{(n)}$ are standard Brownian motions under a $Q$ probability measure with correlations:

$$
\operatorname{Corr}^{\theta}\left(\tilde{W}^{(k)}, \tilde{W}^{(j)}\right)=\theta_{k, j}
$$

We thus obtain a differential equation almost identical to that representing the price of the underlying share with dividend in the standard Black and Scholes model. In fact, the only difference is the addition of the term $-\alpha_{j} \sigma_{j} \rho_{j, j}$ reflecting the quanto characteristic of the option. This term allows the option price to be adjusted to take into account the positive or negative effect of the coverage in terms of the exchange rate. For example, for a given call option, if the correlation between the share and the exchange rate yields is negative, the protection against the exchange rate will have a beneficial effect for the option holder. In this case, the term $-\alpha_{j} \sigma_{j} \rho_{j, j}$ will be positive, thus 
increasing $S_{F}^{(j)}$ and raising the option price. In the case of a positive correlation, the opposite effect will be observed, as the protection against the fluctuation in the exchange rate will act as a penalty. This shows that exchange rate protection is obviously not without consequences. This model allows us to treat cases where the basket contains one or more securities made out in domestic currency as well as cases where several securities share the same currency. For example, if security $k$ and security $j$ have the same currency, we can use $v_{k}=v_{j}$, $\alpha_{k}=\alpha_{j}, Z_{t}^{(k)}=Z_{t}^{(j)}$ and $r_{k}=r_{j}$. The pricing formulas thus remain unchanged. Similarly, if security $k$ is in domestic currency, the model will include one less exchange rate:

$$
\left(C_{0}^{(k)}=1, v_{k}=0, \alpha_{k}=0\right)
$$

As equation 18 is simply a standard geometric Brownian motion, the only strong solution is:

$$
\begin{gathered}
S_{t}^{(j)}=S_{0}^{(j)} \exp \left[\left(r_{j}-\delta_{j}-\alpha_{j} \sigma_{j} \rho_{j, j}-\frac{1}{2} \sigma_{j}^{2}\right) t+\sigma_{j} \widetilde{W}_{t}^{(j)}\right], \\
j=1, \ldots, n .
\end{gathered}
$$

To price a quanto option in our basket, we have to calculate the expected value of the option upon expiry by using equation 20 to represent the price of the underlying securities. The next section deals with the case of the Asian quanto-basket call option.

\section{A. The Asian quanto-basket call option price}

The value of the Asian quanto-basket call option with an exercise price and an expiry date $T$ is given by:

$$
\begin{aligned}
& V_{\text {exact }}=e^{-r T} E^{Q}[\max (A-K, 0)] \\
& =e^{-r T} \int_{-\infty}^{\infty} \max (x-K, 0) f(x) d x,
\end{aligned}
$$


where $f(x)$ is the density function of the arithmetic average, $A$, of the underlying basket value on the predetermined dates $t_{1}, t_{2}, \ldots, t_{m}$. More precisely:

$$
A=\frac{1}{m} \sum_{i=1}^{m} \sum_{j=1}^{n} w_{j} S_{\psi}^{(j)}, \quad 0 \leq t_{1} \leq \ldots \leq t_{m} \leq T
$$

where $\left[w_{j}\right]$ is the vector of the weights of the securities in the basket and where the process for $S^{(j)}$ is given by equation 20 . Notice that in equation 22 , integrating from $-\infty$ to $+\infty$ is equivalent to integrating from 0 to $+\infty$ since $f(x)=0$ for any $x<0$ It should also be noticed that there is no exact solution for expression 21. Indeed, $A$ is a sum of random variables of lognormal distribution. The integral representing the density function of a sum of lognormal random variables, $f(x)$ in this case, cannot be solved analytically. As a result, the price of the option given by equation 22 cannot be directly assessed.

A numerical approximation using a Monte Carlo simulation would allow us to obtain a value that is as accurate as desired. The drawback of this method however is that it requires lengthy calculations. As previously mentioned, analytical approximations have a great deal of value in the financial industry, even if a certain amount of accuracy is lost in the process. In the next sections, we will examine three analytical approximations for our Asian quanto-basket option.

\section{Analytical approximations}

We shall begin this section with the calculation of the first four moments, under the measure $Q$, of the distribution of the arithmetic average of the underlying basket of our options. These moments are used in the different analytical approximations we will examine.

Lemma 1: The first four moments of the arithmetic mean $A$ under the risk neutral measure $Q$ are respectively:

$$
m_{1}^{(f)}=\frac{1}{m} \sum_{i=1}^{m} \sum_{j=1}^{n} w_{j} S_{0}^{(j)} \exp \left[\left(r_{j}-\delta_{j}-\alpha_{j} \sigma_{j} \rho_{j j}\right) t_{i}\right]
$$




$$
\begin{aligned}
& m_{2}^{(f)}=\frac{1}{m^{2}} \sum_{i}^{m} \sum_{i_{2}=1}^{m} \sum_{j_{1}, j_{2}-1}^{n} w_{j_{1}} w_{j_{2}} S_{0}^{\left(j_{1}\right)} S_{0}^{\left(j_{2}\right)} \\
& \times \exp \left[\begin{array}{c}
\left(r_{j_{1}}-\delta_{j_{1}}-\alpha_{j_{1}} \sigma_{j_{1}} \rho_{j_{1} j_{1}}+\sigma_{j_{1}} \sigma_{j_{2}} \theta_{j_{1} j_{2}}\right) t_{\min \left(i_{i} j_{2}\right)} \\
+\left(r_{j_{2}}-\delta_{j_{2}}-\alpha_{j_{2}} \sigma_{j_{2}} \rho_{j_{2}, j_{2}}\right) \xi_{\operatorname{mag}\left(j_{2}\right)}
\end{array}\right], \\
& m_{3}^{(f)}=\frac{1}{m^{3}} \sum_{i}^{m} \sum_{j_{2}, i_{3}-1}^{m} \sum_{j_{1}, j_{2}, j_{3}-1}^{n} w_{j_{1}} w_{j_{2}} w_{j_{3}} S_{0}^{\left(j_{1}\right)} S_{0}^{\left(j_{2}\right)} S_{0}^{\left(j j_{3}\right)} \\
& \times \exp \left[\begin{array}{c}
\left(r_{j}-\delta_{j_{1}}-\alpha_{j_{1}} \sigma_{j_{1}} \rho_{j_{1}, j_{1}}+\sigma_{j_{1}} \sigma_{j_{2}} \theta_{j_{1}, j_{2}}+\sigma_{j_{1}} \sigma_{j_{3}} \theta_{j_{1}, j_{3}}\right) t_{\min \left(i_{1} i_{2}, i_{3}\right)} \\
+\left(r_{j_{2}}-\delta_{j_{2}}-\alpha_{j_{2}} \sigma_{j_{2}} \rho_{j_{2}, j_{2}}+\sigma_{j_{2}} \sigma_{j_{3}} \theta_{j_{2}, j_{3}}\right) t_{\operatorname{med}\left(i_{1}, i_{2}, i_{3}\right)} \\
+\left(r_{j_{3}}-\delta_{j_{3}}-\alpha_{j_{3}} \sigma_{j_{3}} \rho_{j_{3}, j_{3}}\right) t_{\max \left(i_{1}, i_{2} i_{3}\right)}
\end{array}\right], \\
& m_{4}^{(f)}=\frac{1}{m^{4}} \sum_{i_{1}, i_{2}, i_{3}, i_{4}-1}^{m} \sum_{j_{1}, j_{2}, j_{3}, j_{4}-1}^{n} w_{j_{1}} w_{j_{2}} w_{j_{3}} w_{j_{4}} S_{0}^{\left(j_{1}\right)} S_{0}^{\left(j_{2}\right)} S_{0}^{\left(j_{3}\right)} S_{0}^{\left(j_{4}\right)}
\end{aligned}
$$

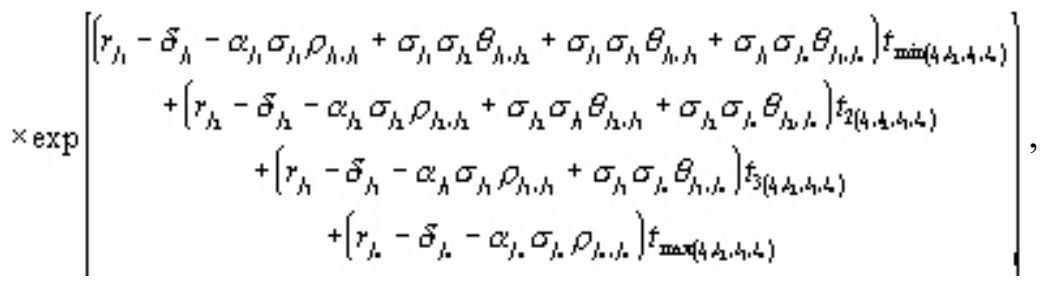

where $x\left(i_{1}, i_{2}, i_{3}, i_{4}\right)$ represents the $x^{\text {th }}$ value of the decreasing ordinate quadruple $\left(i_{1}, i_{2}, i_{3}, i_{4}\right)$.

The demonstration is omitted as it mainly consists of expressing the powers of $A$ in terms of multiple summations and of applying the identity:

$$
E[\exp (a+b Z)]=\exp \left(a+\frac{b^{2}}{2}\right) \text { where } Z \sim N(0,1)
$$


For the next three approximations, we will use the following notation: the expected value as per the density function $g$, is:

$$
\mu^{(g)}=\int_{-\infty}^{\infty} x g(x) d x
$$

and the $k^{\text {th }}$ central moment, in terms of the density function $g$, is:

$$
\psi_{k}^{(g)}=\int_{-\infty}^{\infty}\left(x-\mu^{(g)}\right)^{k} g(x) d x
$$

\section{A. The Lognormal Distribution}

The approximation developed in this section makes use of Edgeworth expansions. Jarrow and Rudd (1982) were the first to suggest using these to solve option pricing problems. The idea consists of using an Edgeworth distribution to replace a candidate density function with an approximate density function having the desired characteristics. In the case of our AQB option, the candidate density function is that of the lognormal sum represented by $f(x)$. As was done by Turnbull and Wakeman (1991) and by Huynh (1994), we chose a lognormal distribution to approximate our sum of lognormals as well as an Edgeworth expansion of the fourth order. Using these general outlines, we can obtain the analytical formula below, allowing us to assess the option price in Appendix 1:

$$
\begin{gathered}
V_{\text {lognomal }}=V_{1}-e^{-r T} \frac{y_{3}^{(f)}-y_{3}^{(a)}}{3 !} \frac{d a}{d x}(K), \\
+e^{-r T} \frac{y_{4}^{(f)}-y_{4}^{(a)}}{4 !} \frac{d^{2} a}{d x^{2}}(K)
\end{gathered}
$$

where

$$
\begin{gathered}
V_{1}=\exp (-\gamma T)\left(\mu_{1} N\left(\hat{d}_{1}\right)-K N\left(\hat{d}_{2}\right)\right), \\
\hat{d}_{1}=\hat{d}_{2}+\hat{b}
\end{gathered}
$$




$$
\begin{gathered}
\hat{d}_{2}=\frac{-\ln (K)+\hat{a}}{\hat{b}}, \\
\hat{a}=2 \ln m_{1}^{(f)}-\frac{1}{2} \ln m_{2}^{(f)}, \\
\hat{b}=\sqrt{\ln m_{2}^{(f)}-2 \ln m_{1}^{(f)}},
\end{gathered}
$$

and $N(\cdot)$ is the distribution function of a standardized normal distribution. It should be noted that the third and fourth moments of the lognormal distribution required to calculate the approximation are respectively:

$$
m_{3}^{(a)}=\left(\frac{m_{2}^{(f)}}{m_{1}^{(f)}}\right)^{3} \text { and } m_{4}^{(a)}=\frac{\left(m_{2}^{(f)}\right)^{6}}{\left(m_{1}^{(f)}\right)^{8}},
$$

where $m_{1}^{(f)}$ and $m_{2}^{(f)}$ are respectively the first two moments of $A$ under the risk neutral measure.

\section{B. The Inverse Gamma Distribution}

The approximation presented in this section is based on the same approach as our lognormal approximation. The only difference lies in the use of an inverse gamma function instead of a lognormal function in the Edgeworth expansion. This choice is based on the work of Milevsky and Posner (1998a). These authors demonstrate that under certain parameter conditions, an infinite sum of correlated lognormal random variable converge asymptotically to an inverse gamma distribution. To compute the price of Asian and basket options, they suggest approximating the distribution of a finite sum of correlated lognormal with an inverse gamma distribution.

In Appendix 2 one can see that the analytical formula to assess the option price is:

$$
V_{\text {gantan }}=V_{2}-\exp (-r T) \frac{y_{3}^{(f)}-y_{3}^{(\bar{g})}}{3 !} \frac{\tilde{g}(K)}{d x}
$$




$$
+\exp (-r T) \frac{y_{4}^{(f)}-y_{4}^{(\bar{g})}}{4 !} \frac{d^{2} \tilde{g}(K)}{d x^{2}},
$$

where $g$ is the density function of a random variable of the inverse gamma distribution,

$$
\begin{gathered}
V_{2}=\exp (-r T) \mu_{1} \mathbf{G}\left(\frac{1}{K} \mid \alpha-1, \beta\right)-K \mathrm{G}\left(\frac{1}{K} \mid \alpha, \beta\right), \\
\alpha=\frac{-2 m_{2}^{(f)}+\left(m_{1}^{(f)}\right)^{2}}{-m_{2}^{(f)}+\left(m_{1}^{(f)}\right)^{2}}, \\
\beta=\frac{m_{2}^{(f)}-\left(m_{1}^{(f)}\right)^{2}}{m_{1}^{(f)} m_{2}^{(f)}}
\end{gathered}
$$

and $\mathbf{G}(\cdot \mid \eta, \lambda)$ is the distribution function of a gamma distribution with the parameters $(\eta, \lambda)$. It should be noted that the third and fourth moments of the inverse gamma distribution required to calculate the approximation are respectively:

$$
m_{3}^{(\bar{g})}=\frac{m_{1}^{(f)}\left(m_{2}^{(f)}\right)^{2}}{2\left(m_{1}^{(f)}\right)^{2}-m_{2}^{(f)}}
$$

and

$$
m_{4}^{(\bar{s})}=\frac{\left(m_{1}^{(f)}\right)^{2}\left(m_{2}^{(f)}\right)^{3}}{2\left(m_{2}^{(f)}\right)^{2}-7\left(m_{1}^{(f)}\right)^{2} m_{2}^{(f)}+6\left(m_{1}^{(f)}\right)^{4}},
$$

where $m_{1}^{(f)}$ and $m_{2}^{(f)}$ are respectively the first two moments of $A$ under the risk neutral measure. 


\section{The Johnson distribution}

The idea of this approach is again to replace the true density function with an approximate density. Recall that the goal is to compute the integral of equation 22:

$$
\begin{aligned}
V_{\text {exad }} & =e^{-r T}\left(\int_{-\infty}^{\infty}(x-K) f(x) d x-\int_{-\infty}^{X}(x-K) f(x) d x\right) \\
& =e^{-r T}\left(E^{Q}[A]-X-\int_{-\infty}^{X}(x-K) f(x) d x\right) \\
& =e^{-r T}\left(E^{Q}[A]-K+\int_{-\infty}^{X}\left(\int_{-\infty}^{x} f(y) d y\right) d x\right),
\end{aligned}
$$

where the last equality is obtain from integration by parts. In this case, the true density $f$ is replaced by an approximating density chosen among the Johnson family which is a collection of statistical distributions parameterized by four quantities. More precisely, if $Z$ represents a standard normal random variable, then the random variable $X$ where:

$$
X=\gamma+\delta \phi^{-1}\left(\frac{z-\alpha}{\beta}\right),
$$

has a Johnson distribution. The function $\phi$ is usually restricted to $\ln (\cdot)$ (Type I) or to $\sinh ^{-1}(\cdot)$ (Type II). ${ }^{2}$ The four parameters are determined to match the first four moments of the original distribution. Therefore:

$$
V_{\text {dohwo }}=e^{-r T}\left(E^{Q}[A]-K-\int_{-\infty}^{X}\left(\int_{-\infty}^{x} h(y) d y\right) d x\right),
$$

where $h(\cdot)$ is the Johnson density function. Using the type II distribution, Posner and Milevsky (1998) have shown that:

2. Since $\sinh (x)=\frac{e^{x}-e^{-x}}{2}$, one can show that $\sinh ^{-1}(x)=\ln \left[x+\sqrt{x^{2}+1}\right]$. 


$$
\begin{gathered}
\int_{-\infty}^{X}\left(\int_{-\infty}^{x} h(y) d y\right) d x=(K-\gamma) N(q) \\
+\frac{\delta}{2} \exp \left(\frac{1}{2 \beta^{2}}\right)\left[\exp \left(\frac{\alpha}{\beta}\right) N\left(q+\frac{1}{\beta}\right)-\exp \left(-\frac{\alpha}{\beta}\right) N\left(q-\frac{1}{\beta}\right)\right]
\end{gathered}
$$

with

$$
q=\alpha+\beta \sinh ^{-1}\left(\frac{K-\gamma}{\delta}\right)
$$

To determine the values of $\gamma, \delta, \alpha$ and $\beta$ which match the first four moments of the original distribution, we use the numerical algorithm given in Hill, Hill and Holder (1976).

\section{Performance of the Analytical Approximations}

To establish the numerical accuracy of our three analytical approximations, two simulation experiments are conducted. The first examines options priced using our analytical approximations and compares the results of the approximations with those of Monte Carlo simulations using 1,000,000 trajectories. Although the parameters used in this experiment (volatilities, correlations, etc.) take on real values, they nevertheless remain somewhat arbitrary. Indeed, the AQB option depends on large number of different parameters. Examining individually the performance of the approximations on all the possible parameter combinations would be a very difficult task. We will thus conduct a second simulation experiment prompted by Broadie and Detemple (1996), which uses random parameters generated from a realistic set of values. The prices obtained will also be compared to prices derived again from precise Monte Carlo simulations. The following paragraphs provide the details of these numerical experiments.

Table 2 presents the basic values used to perform the first simulation experiment. ${ }^{3}$ For each example, we employed these values with the

3. The values for the stock return volatilities, exchange rate volatilities, correlations between the stock returns and exchange rates and the correlations between stock returns were 
TABLE 2. Parameter Values Used in Tables 3 and 4

\begin{tabular}{|c|c|c|c|c|}
\hline \multicolumn{3}{|c|}{ Parameter(s) } & \multicolumn{2}{|c|}{ Value(s) } \\
\hline \multicolumn{3}{|c|}{ Number of securities in the basket } & \multicolumn{2}{|l|}{5} \\
\hline \multicolumn{3}{|c|}{ Initial stock price } & \multicolumn{2}{|c|}{$50 ; 55 ; 18 ; 35 ; 70$} \\
\hline \multicolumn{3}{|c|}{ Stock prices volatilities } & \multicolumn{2}{|c|}{$0.17 ; 0.29 ; 0.24 ; 0.24 ; 0.35$} \\
\hline \multicolumn{3}{|c|}{ Dividend rates } & \multicolumn{2}{|c|}{$0.03 ; 0.03 ; 0.03 ; 0.03 ; 0.03$} \\
\hline \multicolumn{3}{|c|}{ Exchange rate volatilities } & \multicolumn{2}{|c|}{$0.14 ; 0.07 ; 0.14 ; 0.12 ; 0.15$} \\
\hline \multicolumn{3}{|c|}{$\begin{array}{l}\text { Correlations between the stock price } \\
\text { and exchange rates }\end{array}$} & \multicolumn{2}{|c|}{$0.07 ; 0.15 ; 0.04 ; 0.13 ; 0.29$} \\
\hline \multicolumn{3}{|c|}{ Domestic risk-free rate } & \multicolumn{2}{|l|}{0.06} \\
\hline \multicolumn{3}{|c|}{ Foreign risk-free rate } & \multicolumn{2}{|c|}{$0.05 ; 0.05 ; 0.05 ; 0.05 ; 0.05$} \\
\hline \multicolumn{3}{|c|}{ Weights } & \multicolumn{2}{|c|}{$0.1 ; 0.3 ; 0.2 ; 0.18 ; 0.22$} \\
\hline \multicolumn{3}{|c|}{ Maturities (days) } & \multicolumn{2}{|c|}{$30 ; 90 ; 180 ; 270$} \\
\hline \multicolumn{3}{|c|}{ Average } & \multicolumn{2}{|c|}{ Monthly } \\
\hline \multicolumn{3}{|c|}{ Strike price (in proportion of the initial basket value) } & \multicolumn{2}{|l|}{1.00} \\
\hline 1.00 & 0.11 & 0.40 & 0.38 & 0.16 \\
\hline 0.11 & 1.00 & 0.11 & 0.00 & 0.45 \\
\hline \multicolumn{5}{|c|}{ correlations between stock prices } \\
\hline 0.40 & 0.11 & 1.00 & 0.24 & 0.15 \\
\hline 0.38 & 0.00 & 0.24 & 1.00 & 0.06 \\
\hline 0.16 & 0.45 & 0.15 & 0.06 & 1.00 \\
\hline
\end{tabular}

exception of an analyzed parameter that we used as a variable. Tables 3 and 4 present the numerical results. In table 3 , we used the volatility of the basket as well as the term of the option as variables. The high and low volatilities respectively correspond to an increase and a decrease of $50 \%$ of the basic volatilities. The results from this table reveal that the accuracy of the lognormal approximation deteriorates with increases in volatility and term length. The inverse gamma and Johnson distributions proved to be very good for all situations presented in this table. Table 4 shows the results obtained for different exercise prices. As in table 3, the values of the parameters used are those of the basic basket. Although not reported here, we also examined the effects of other parameters (exchange rate volatility, number of shares in the basket, correlation between shares and exchange rate, etc.). The results obtained were similar to those reported in table 3 and 4.

Finally, to establish the accuracy of the approximations in a more

obtained using stock index and exchange rate data from the Datastream database for the following countries: Australia, Canada, Japan, New Zealand, and Mexico. All exchange rates are expressed in terms of local currency per U.S. dollar. 
TABLE 3. Asian Quanto-basket Call Option Prices

\begin{tabular}{|c|c|c|c|c|}
\hline $\begin{array}{l}\text { Basket } \\
\text { Volatility }\end{array}$ & Sim & Lnorm & Gamma & Johnson \\
\hline \multicolumn{5}{|c|}{30 days to maturity } \\
\hline low & 0.5615 & 0.5626 & 0.5625 & 0.5626 \\
\hline average & 1.0835 & 1.0846 & 1.0853 & 1.0856 \\
\hline high & 1.6037 & 1.6045 & 1.6077 & 1.6087 \\
\hline \multicolumn{5}{|c|}{90 days to maturity } \\
\hline low & 0.7207 & 0.7202 & 0.7200 & 0.7200 \\
\hline average & 1.3598 & 1.3575 & 1.3604 & 1.3603 \\
\hline high & 2.0013 & 1.9890 & 2.0012 & 2.0008 \\
\hline \multicolumn{5}{|c|}{180 days to maturity } \\
\hline low & 0.9365 & 0.9372 & 0.9371 & 0.9368 \\
\hline average & 1.7337 & 1.7264 & 1.7346 & 1.7334 \\
\hline high & 2.5236 & 2.4995 & 2.5344 & 2.5302 \\
\hline \multicolumn{5}{|c|}{270 days to maturity } \\
\hline low & 1.1163 & 1.1199 & 1.1197 & 1.1192 \\
\hline average & 2.0376 & 2.0252 & 2.0407 & 2.0378 \\
\hline high & 2.9585 & 2.8995 & 2.9661 & 2.9565 \\
\hline
\end{tabular}

Note: Sim is Monte Carlo simulation with 1,000,000 sample paths. Lnorm is the lognormal approximation. Gamma is the gamma-inverse approximation and Johnson is the Johnson approximation.

TABLE 4. Asian Quanto-basket Call Option Prices

\begin{tabular}{lllll}
\hline Strike & Sim & Lnorm & Gamma & Johnson \\
\hline 30 days to maturity & & & & \\
0.95 & 2.6223 & 2.6351 & 2.6244 & 2.6230 \\
1.00 & 1.0835 & 1.0846 & 1.0853 & 1.0856 \\
1.05 & 0.3092 & 0.3032 & 0.3129 & 0.3113 \\
90 days to maturity & & & & \\
0.95 & 2.8177 & 2.8321 & 2.8159 & 2.8160 \\
1.00 & 1.3598 & 1.3575 & 1.3604 & 1.3603 \\
1.05 & 0.5267 & 0.5105 & 0.5268 & 0.5266 \\
180 days to maturity & & & \\
0.95 & 3.1001 & 3.1238 & 3.1034 & 3.1006 \\
1.00 & 1.7337 & 1.7264 & 1.7346 & 0.8549 \\
1.05 & 0.8516 & 0.8270 & 0.8540 & \\
270 days to maturity & & & 3.3457 \\
0.95 & 3.3395 & 3.3709 & 3.3515 & 2.0378 \\
1.00 & 2.0376 & 2.0252 & 2.0407 & 1.1415 \\
1.05 & 1.1417 & 1.1031 & 1.1404 & \\
\hline
\end{tabular}

Note: Strike is the initial basket value divided by the strike price. Sim is Monte Carlo simulation with 1,000,000 sample paths. Lnorm is the lognormal approximation. Gamma is the gamma-inverse approximation and Johnson is the Johnson approximation. 
TABLE 5. Parameter Choices for the Test Pool of Options

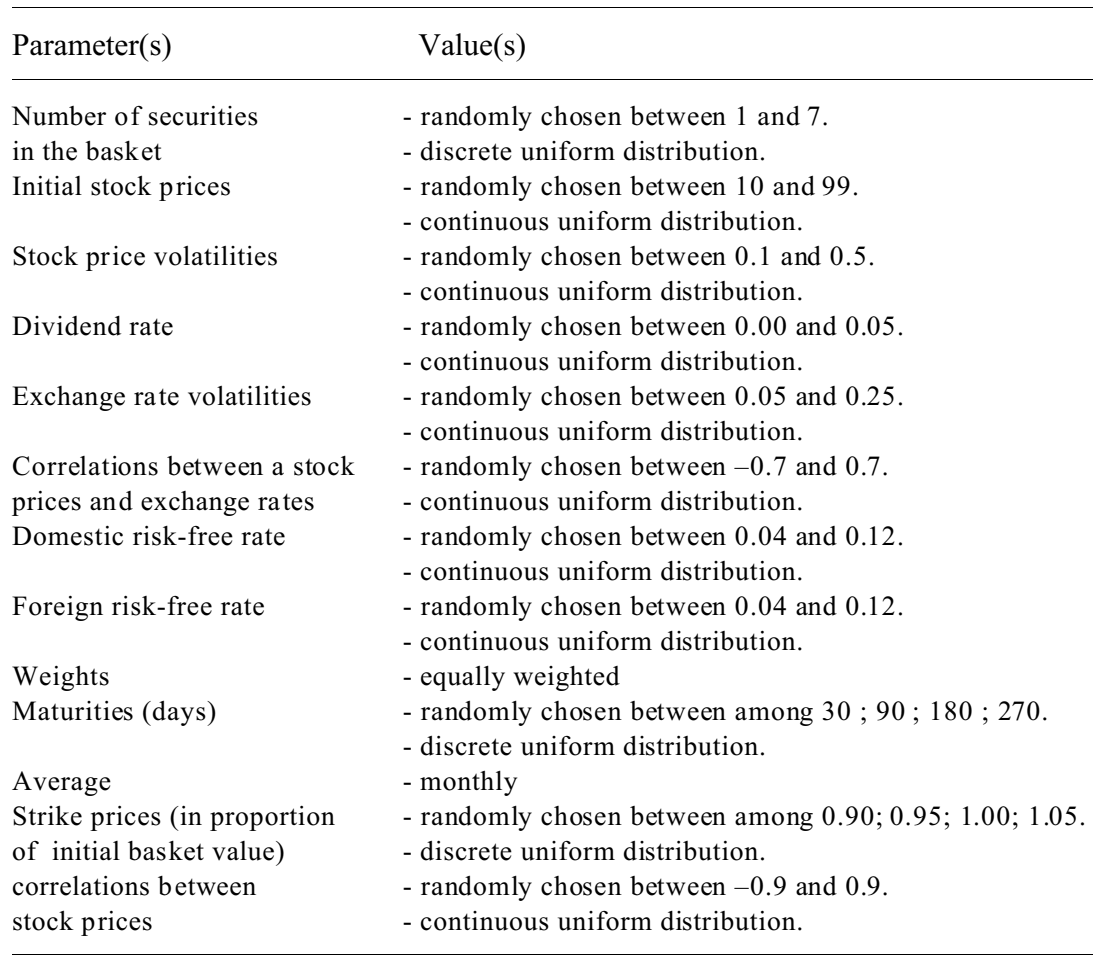

general way, we perform an analysis similar to that used by Broadie and Detemple (1996). The method consists of randomly choosing the parameters of a large test pool of options within a set of realistic values. The options are then priced using our analytical approximations and the obtained values are compared to those of a Monte Carlo simulation. Table 5 describes the distributions used to obtain the options parameters. Note that the correlation matrices that are not positive definite are rejected. We will measure the aggregate error by the sum of the root mean square of individual errors (RMS). More precisely, we will have:

$$
\operatorname{RMS}(m)=\sqrt{\frac{1}{m} \sum_{i=1}^{m} e_{i}^{2}},
$$

where $m$ is the number of options included in the study, 


$$
e_{i}=\frac{\left|C_{i}(a)-C_{i}\right|}{C_{i}}
$$

$C_{i}(a)$ is the price given by the analytical approximation for option $i$ and $C_{i}$ is the price given by the Monte Carlo simulation $(500,000$ trajectories) for the option $i$. We fixed the value of $m$ at 5,000 and eliminate the cases where $C_{i}<0.50$ to avoid the occurrence of too many relative errors due to a low divisor. We also eliminate the cases for which the non-linear algorithm for the determination of the parameters required by the Johnson's approximation failed to converge (18 cases). The total number of valid cases after these deletions is 4205 .

For the lognormal approximation, we obtained a RMS $=0.0183$ as well as 113 options with a relative error of more than 5\%. This means that $97.3 \%$ of the options priced using the lognormal approximation had a relative error below $5 \%$. The maximum relative error was of $25.1 \%$. In the case of the inverse gamma approximation, we obtained a RMS $=$ 0.0071 as well as 5 options with a relative error of more than $5 \% .99 .8 \%$ of options priced using the inverse gamma approximation had thus a relative error of less than $5 \%$. The maximum relative error was of $6.98 \%$. Finally, for the Johnson approximation, a RMS of 0.0018 was obtained. All options were computed with an error bellow 5\%. A detailed analysis of the results revealed that the most important factors for explaining the pricing errors were large values for the maturity and basket volatility.

In addition, we have also examined the precision of the delta associated with each method. These quantities are computed numerically. More precisely, for each pricing method, we add a small quantity $\varepsilon>0$ to each security included in the basket: $S_{0}^{(j)}+\varepsilon$. We obtain a new approximation for the option price denoted $V_{a y s}^{(x)}$ and compute numerically the first derivative with:

$$
V_{a p y}^{\prime}=\frac{V_{a p y}^{(s)}-V_{a y y}}{\varepsilon}
$$

The results for these quantities are in line with those obtained for the prices. The RMS for the lognormal, gamma and Johnson are 0.0306, 0.0128 and 0.0061 respectively. Again, a detailed analysis showed that the large errors are mostly associated with large maturities or large 
basket volatility.

In summary, the analysis reveals that the Johnson approximation is the most precise method and should be preferred to the lognormal and gamma inverse approximations. It should be pointed out, however, that the convergence associated with the non-linear algorithm required by this approximation should be monitored closely. Although a failure to converge does not occur frequently, it is still a possibility that might lead to substantial errors if undetected. Our second choice is the gamma inverse approximation which also shows very good results that are well within the precision required by many practical situations.

\section{Conclusion}

The Asian quanto-basket option is a contract allowing an investor to participate to the return of an industrial or economic sector with an international exposure without the foreign exchange risk exposition. Since the payoffs of these contracts involve sums of lognormal random variables, no analytical solution is available to compute its price. We have thus examined here the precision of three different analytical approximations which can be computed in fractions of seconds for the Asian quanto-basket option. Using a large test pool of options, the Johnson distribution was found to be the most accurate approximation followed by the inverse gamma. For both approximations, the magnitude of the pricing errors appears to be well within the tolerance level required by many practical applications.

\section{Appendix Analytical Approximations}

Appendix 1. Lognormal Approximation

This section provides the derivations associated with the results presented in section 4A. Let $a(x)$ be the lognormal density of the random variable $\exp (\hat{a}+\hat{b} Z)(Z-N(0,1))$ which is chosen as an approximation for $f(x)$. We can show (see Jarrow and Rudd [1982, p. 366]) that $f(x)$ can be written as the following Edgeworth expansion:

$$
f(x)=a(x)+\frac{\psi_{1}^{(5)}-\psi_{2}^{(t)}}{2 !} \frac{d^{2} a(x)}{d x^{2}}-\frac{\left.\psi_{3}^{(J)}-\psi_{3}^{(t)}\right)}{3 !} \frac{d^{3} a(x)}{d x^{3}}
$$




$$
+\frac{\left(\psi_{+}^{(J)}-\psi_{+}^{(+)}\right)+\left[3\left(\psi_{2}^{(+)}\right)^{2}-3\left(\psi_{2}^{(J)}\right)^{2}\right]+3\left(\psi_{2}^{(J)}-\psi_{2}^{(+)}\right)^{2}}{4 !} \frac{d^{+} a(x)}{d x^{4}}+s(x)
$$

where the centered moments $\psi_{1}^{(*)}$ are defined in equation 30 and $\varepsilon(x)$ is an error term. In general, no analytical behavior can be attached to this error term. Since a lognormal distribution is entirely described by its first two moments, we choose function $a(x)$ such that the first two moments are identical to those of $f(x)$. Hence, $\hat{a}$ and $\hat{b}$ must be equal to the expressions given in equations 35 and 36 . We can thus rewrite equation 49 as follows:

$$
f(x)=a(x)-\frac{\psi_{3}^{(5)}-\psi_{3}^{(t)}}{3 !} \frac{d^{3} a(x)}{d x^{3}}+\frac{\psi_{4}^{(f)}-\psi_{4}^{(t)}}{4 !} \frac{d^{4} a(x)}{d x^{4}}+s(x)
$$

We define the third approximation as $V_{\text {lognormal }}$ :

$$
\begin{aligned}
& e^{-r T} \int_{-\infty}^{\infty} \max (x-K, 0) f(x) d x \\
& \cong e^{T r} \int_{-\infty}^{\infty} \max (x-K, 0)\left[a(x)-\frac{y_{3}^{(r)}-y_{3}^{(0)}}{3 !} \frac{d^{3} a(x)}{d x^{3}}\right. \\
& \left.+\frac{y_{4}^{(5)}-y_{4}^{(0)}}{4} \frac{d^{4} a(x)}{d x^{4}}+E(x)\right] d x \\
& =e^{-r T} \int_{K}^{\infty}(x-K)\left[a(x)-\frac{\psi_{3}^{(S)}-\psi_{3}^{(t)}}{3 !} \frac{d^{3} a(x)}{d x^{3}}+\frac{\psi_{4}^{(5)}-\psi_{4}^{(t)}}{4 !} \frac{d^{4} a(x)}{d x^{4}}+s(x)\right] d x \\
& =V_{\text {lognormal }} \text {. }
\end{aligned}
$$

Using the following identity

$$
\int_{K}^{\infty}(x-K) \frac{d^{\prime} a(x)}{d x^{\prime}} d x=\frac{d^{j-2} a}{d x^{1-2}}(K) \text { for } j \geq 2
$$

(see Jarrow and Rudd, 982, p. 355), we obtain:

$$
V_{\text {ap } 33}=e^{-r \tau} \int_{K}^{\infty}(x-K) a(x) d x
$$




$$
-e^{-r \tau} \frac{\psi_{3}^{(J)}-\psi_{3}^{(t)}}{3 !} \frac{d a}{d x}(K)+e^{-r \tau} \frac{\psi_{4}^{(J)}-\psi_{4}^{(t)}}{4 !} \frac{d^{2} a}{d x^{2}}(K)
$$

The first term in this equation if the Black-Scholes formula since the integration is performed with respect to the lognormal density. The centered moments of the lognormal distribution are easily obtained from the first four moments of . Hence,

$$
\begin{aligned}
& m_{1}^{\left({ }^{\prime}\right)}=\exp \left(\hat{a}+\frac{\hat{b}^{2}}{2}\right)=m_{1}^{(J)} \\
& m_{1}^{(+)}=\exp \left(2 \hat{a}+2 \hat{b}^{2}\right)=m_{1}^{(f)}, \\
& m_{3}^{(+)}=\exp \left(3 \hat{a}+\frac{9 \hat{b}^{2}}{2}\right)=\left(\frac{m_{2}^{(+)}}{m_{1}^{(+)}}\right)^{3}=\left(\frac{m_{2}^{(f)}}{m_{1}^{(f)}}\right)^{3}, \\
& m_{+}^{(+)}=\exp \left(4 \hat{a}+8 \hat{b}^{2}\right)=\frac{\left(m_{2}^{(+)}\right)^{6}}{\left(m_{1}^{(+)}\right)^{8}}=\frac{\left(m_{2}^{(f)}\right)^{6}}{\left(m_{1}^{(f)}\right)^{8}} .
\end{aligned}
$$

Those associated with $f$ can be found from lemma 1 .

Appendix 2. Gamma-inverse Approximation

This section provides the derivations associated with the results presented in section $4 \mathrm{~B}$. As for the lognormal distribution, the gamma-inverse is entirely described by its first two moments. We thus choose the gamma-inverse function $\tilde{g}(x)$ such that the first two moments are identical to those of $f(x)$. Because of this, our approximation will be defined in a similar fashion as the one provided in Appendix 1, that is:

$$
\begin{aligned}
& V_{\text {gormex }}=e^{-r \tau} \int_{-\infty}^{\infty} \max (x-K, 0)\left[g_{\delta}(x)-\frac{y_{3}^{(f)}-y_{3}^{[\overline{8})}}{3 !} \frac{d^{3} \tilde{g}(x)}{d x^{3}}\right. \\
& \left.+\frac{w_{4}^{(j)}-w_{4}^{[\overline{8})}}{4 !} \frac{\left.d^{4} \tilde{g}(x)\right]}{d x^{4}}\right] d x
\end{aligned}
$$


Definition 2: The density function of a gamma random variable with parameters $(\alpha, \beta), X \sim G(\alpha, \beta)$, is given by:

$$
g_{n}(x)=\frac{p^{-\alpha} x^{\alpha-1} \exp \left(-\frac{\gamma}{\sigma}\right)}{\Gamma(\alpha)}, \forall x>0
$$

where $\alpha>0, \beta>0$ and $\Gamma(\alpha)$ is the gamma function.

Proposition 3: The density function of the random variable $Y=1 / x$ where $X \sim G(\alpha, \beta)$ is given by:

$$
\tilde{g}(y)=\frac{\beta^{-\alpha}}{y^{\alpha+1} \Gamma(\alpha)} \exp \left(-\frac{1}{y \beta}\right), \forall y>0 .
$$

We will then say that $Y$ will have a gamma-inverse distribution written as $X \sim G(\alpha, \beta)$.

Proposition 4. Let $X \sim G(\alpha, \beta)$. The moments of $Y$ are given by:

$$
E\left[Y^{i}\right]=\frac{1}{\beta(\alpha-1)(\alpha-2) \ldots(\alpha-i)}
$$

where $i<\alpha$.

We thus have to choose a gamma-inverse distribution such that its first two moments will be equal to the first two moments of $A$. Let

$$
\alpha=\frac{-2 m_{1}^{(5)}+\left(m_{1}^{(j)}\right)^{2}}{-m_{2}^{(J)}+\left(m_{1}^{(J)}\right)^{2}} \text { and } \beta=\frac{m_{1}^{(j)}-\left(m_{1}^{(j)}\right)^{2}}{m_{1}^{(J)} m_{2}^{(J)}}
$$

Since

$$
\int_{x}^{\infty}(x-K) \frac{d^{\prime} \tilde{g}(x)}{d x^{\prime}} d x=\frac{d^{\prime-2} \tilde{g}(K)}{d x^{-2}} \text { for } j \geq 2,
$$

then

$$
\begin{gathered}
V_{\text {Eummt }}=e^{-r r} \int_{K}(x-K) \tilde{g}(x) d x-e^{-r r} \frac{\psi_{3}^{(J)}-\psi_{3}^{[\bar{g}]}}{3 !} \frac{d \tilde{g}}{d x}(K) \\
+e^{-r \tau} \frac{\psi_{t}^{(J)}-\psi_{t}^{[\bar{g}]}}{4 !} \frac{d^{2} \tilde{g}}{d x^{2}}(K) .
\end{gathered}
$$


Using the change of variable $y=1 / X$, we can rewrite the first term as:

$$
e^{-r \tau} \int_{K}^{\infty}(x-K) \tilde{g}(x) d x=e^{-r \tau}\left(\mu_{1} \mathbf{G}\left(\frac{1}{K} \mid \alpha-1, \beta\right)-K \mathbf{G}\left(\frac{1}{K} \mid \alpha, \beta\right)\right)
$$

where $G(\cdot \mid \eta, \lambda)$ is the distribution function of a gamma with parameters $(\eta, \lambda)$. Finally, the approximation uses the first four moments of the gamma-inverse distribution. By construction, its first two moments are identical to those of $Y$.

We thus derive here the third and fourth moments of $Y$ :

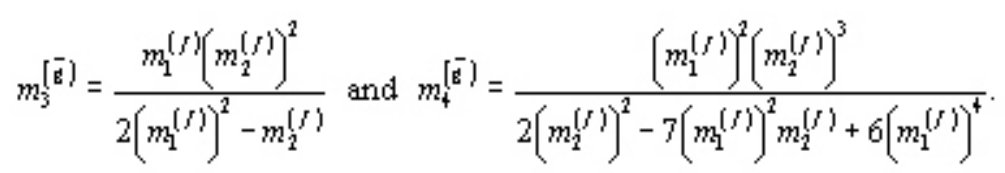

\section{References}

Bakshi, G., and Madan. D. 2002. Average rate claims with emphasis on catastrophe loss options. Journal of Financial and Quantitative Analysis 37:93-115.

Black F., and Scholes M. 1973. The pricing of options and corporate liabilities. Journal of Political Economy 81:637-654.

Broadie M., and Detemple, J. 1996. American option valuation: New bounds, approximations and a comparison of existing methods. Review of Financial Studies 9:1211-1250.

Curran, M. 1994. Valuing Asian and portfolio options by conditioning on the geometric mean. Management Science 40:1705-1711.

Dufresne, D. 2000. Laguerre series for Asian and other options. Mathematical Finance 10:407-428.

Fu, M.; Madan, D.; and Wang, T. 1999. Pricing Asian options: A comparison of analytical and Monte Carlo methods. Journal of Computational Finance 2:49-74.

Geman, H., and Yor, M. 1993. Bessel processes, Asian options and perpetuities. Mathematical Finance 3:349-375.

Gentle D. 1993. Basket W eaving. Risk 6:51-52.

Harrison M., and Pliska S. 1981. Martingales and stochastic integrals in the theory of continuous trading. Stochastic Processes and their Applications 11:215-260.

Hill, I.; Hill, R.; and Holder, R. 1976. Fitting Johnson curves by moments. Applied Statistics 25:180-192.

Hull, J., and White, A. 1993. Efficient procedures for valuing European and American path-dependent options. Journal of Derivatives 1:21-31.

Huynh C. B. 1994. Back to baskets. Risk, 7:59-61. 
Jarrow R., and Rudd, A. 1982. Approximate option valuation for arbitrary stochastic processes. Journal of Financial Economics 10:347-369.

Joy, C.; Boyle, P.; and T ang, K. 1996. Quasi Monte Carlo methods in numerical finance. Management Science 42:926-938.

Kemna, A., and Vorst, T. 1990. A pricing method for options based on average asset values. Journal of Banking and Finance 14:113-129.

Levy, E. 1992. The valuation of average rate currency options. Journal of International Money and Finance 11:474-491.

Milevsky M., and Posner, S. 1998a. Asian options, the sum of lognormals and the reciprocal Gamma distribution. Journal of Financial and Quantitative Analysis 33:409-422.

Milevsky M., and Posner, S. 1998b. A closed-form approximation for valuing basket options. Journal of Derivatives 6:54-61.

Milevsky, M., and Posner, S. 1999. Another moment for the average option. Derivatives Quarterly (Summer):47-53.

Nielsen, J. and Sandmann, K. 2002. Pricing bounds on Asian options. Forthcoming in Journal of Financial and Quantitative Analysis.

Posner, S., and Milevsky, M. 1998. Valuing exotic options by approximating the SPD with higher moments. Journal of Financial Engineering 7:109125 .

Reiner E. 1992. Quanto mechanics. Risk 5:59-63.

Rogers, L., and Shi, Z. 1995. The value of an Asian option. Journal of Applied Probability 32:1077-1088.

Rubinstein, M. 1994. Return to Oz. Risk 7:67-71.

Turnbull, S., and Wakeman, L. 1991. A quick algorithm for pricing European average options. Journal of Financial and Quantitative Analysis 26:377389.

Vecer, J. 2001. A new PDE approach for pricing arithmetic average Asian options. Journal of Computational Finance 4:105:113.

Vorst, T. 1992. Prices and hedge ratios of average exchange rate options. International Review of Financial Analysis 1:179-193. 